\title{
Track Xplorer: A System for Visual Analysis of Sensor-based Motor Activity Predictions
}

\author{
Marco Cavallo ${ }^{1}$ and Çă̆atay Demiralp ${ }^{1}$
}

${ }^{1}$ IBM Research

\begin{abstract}
With the rapid commoditization of wearable sensors, detecting human movements from sensor datasets has become increasingly common over a wide range of applications. To detect activities, data scientists iteratively experiment with different classifiers before deciding which model to deploy. Effective reasoning about and comparison of alternative classifiers are crucial in successful model development. This is, however, inherently difficult in developing classifiers for sensor data, where the intricacy of long temporal sequences, high prediction frequency, and imprecise labeling make standard evaluation methods relatively ineffective and even misleading.

We introduce Track Xplorer, an interactive visualization system to query, analyze, and compare the predictions of sensor-data classifiers. Track Xplorer enables users to interactively explore and compare the results of different classifiers, and assess their accuracy with respect to the ground-truth labels and video. Through integration with a version control system, Track Xplorer supports tracking of models and their parameters without additional workload on model developers. Track Xplorer also contributes an extensible algebra over track representations to filter, compose, and compare classification outputs, enabling users to reason effectively about classifier performance. We apply Track Xplorer in a collaborative project to develop classifiers to detect movements from multisensor data gathered from Parkinson's disease patients. We demonstrate how Track Xplorer helps identify early on possible systemic data errors, effectively track and compare the results of different classifiers, and reason about and pinpoint the causes of misclassifications.
\end{abstract}

\section{Introduction}

The extensive diffusion of consumer-level wearable devices has opened up many possibilities for activity monitoring and analysis. Fitness trackers, from smart watches to wristbands, are increasingly used by people to track their daily activities. Similarly, a wide variety of biosensors is starting to play an important role in continuous outpatient monitoring. Detecting motor activities (i.e., limb movements) from sensor data and understanding their normal and abnormal variation has a great potential to inform and predict the well-being of healthy persons and patients alike. To this end, developing classifiers that successfully detect different

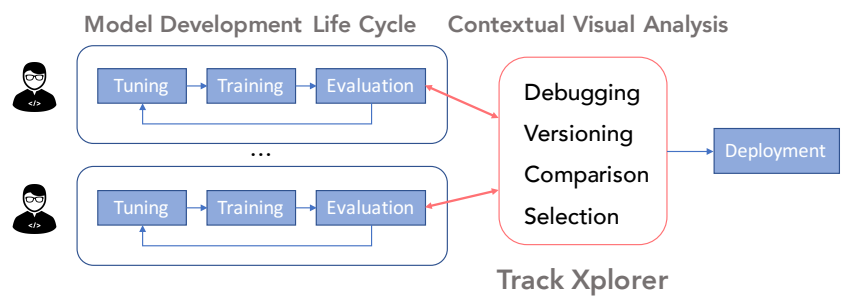

Figure 1: Improving model development life cycle. Track Xplorer enhances the comparative evaluation of multiple predictive models through contextual visual analysis. types of physical motion from sensor data is critical for the reliability of insights derived from any downstream analysis.

Classifier development is an iterative process in which data scientists start with some alternative models and associated hyperparameters, train the models on a subset of the available data, and then evaluate their performance on test data. During the evaluation step, data scientists use aggregate performance metrics such as accuracy score, precision and recall to establish how well a classifier predicts specific activities. Data scientists then tune their algorithms and parameters using these insights, restarting the cycle and iteratively improving their models. Analyzing the output of predictive models is a crucial step in the life cycle of iterative model development (Fig. 1). Although aggregate evaluation metrics can be useful for overall performance assessment and reporting, they don't carry sufficient detail to facilitate interpretability and drill-down analysis needed to debug and fine-tune the models. Aggregate metrics treat predictions uniformly, hiding differences within them. Researchers have recently proposed interactive tools (e.g., [ACD*15, $\left.\left.\mathrm{AHH}^{*} 14, \mathrm{RAL}^{*} 17\right]\right)$ to help evaluate classifier performances both in aggregate and at instance level, primarily using interactive histograms of instance class probabilities.

When evaluating motor activity classifiers, the inadequacy of aggregate metrics becomes even more pronounced due to the intricate temporal nature of data that classifiers operate on. Each activity and its time window is recognized by considering multiple model predictions computed at high frequency, and is often validated against labels generated through manual video annotation. The need to evaluate a classifier's output against 


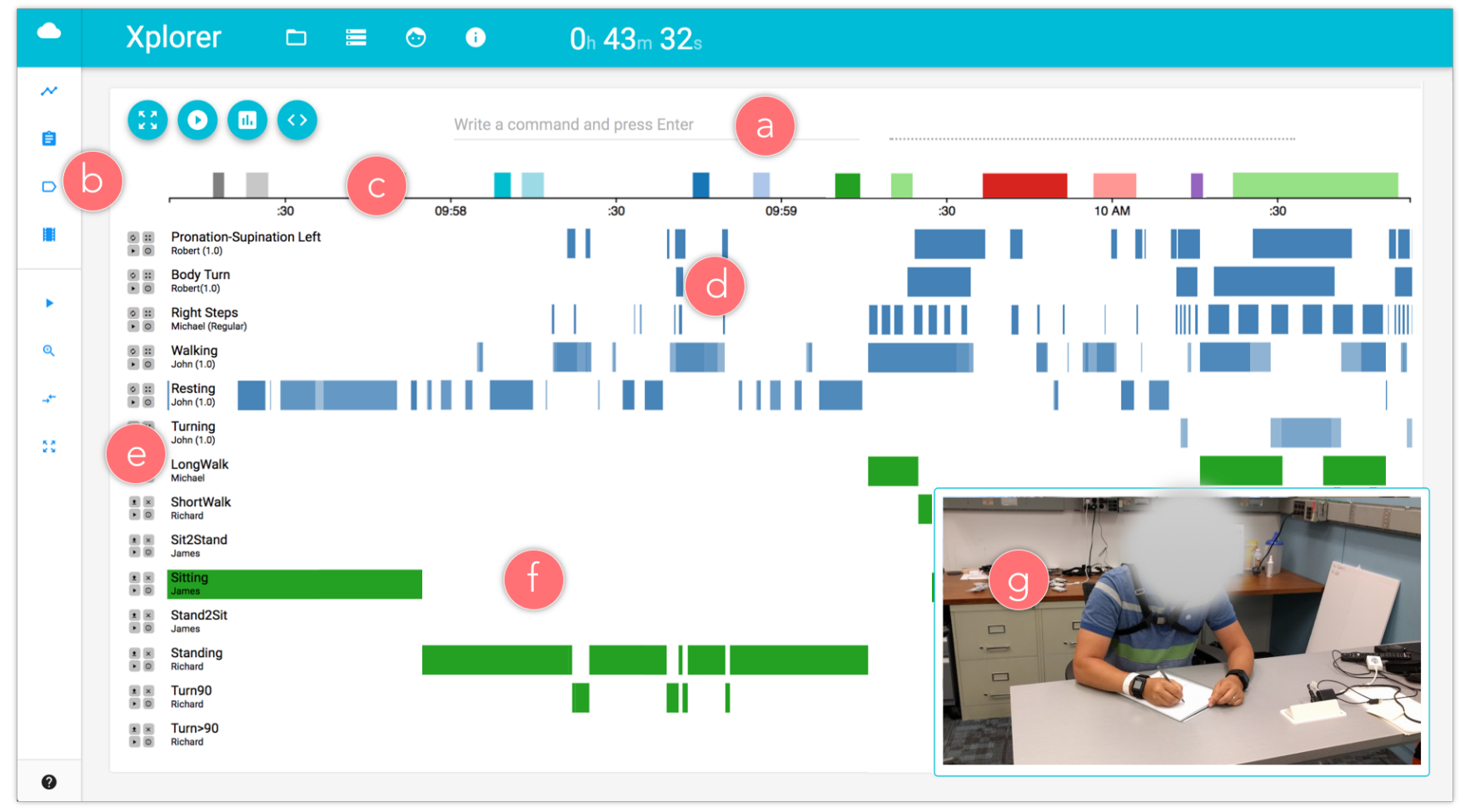

Figure 2: Track Xplorer interface. The main view shows a timeline for a set of stacked linear tracks. A track can represent the sequence of event predictions generated by a classifier ( $d$, in blue) or the series of annotations used as ground-truth labels (f, in green). Tracks are associated to the predicted motor activity, to the name of the person who generated the results or labels and to the model version (in case of classifiers). This information is displayed at the left margin of each track (e), together with four buttons that enable performing track operations. A dedicated protocol track (c) can be used to easily navigate to regions of interest, while a synchronized video player $(\mathrm{g})$ lets users validate the context of event predictions. Users can customize the appearance of tracks through a collapsible sidebar on the left (b). A command-line interface (a) lets users run commands from Track Xplorer's command set, which also supports the operations of a visual track algebra.

manual labels that are generally affected by human interpretation, goal and bias further limits the effectiveness of aggregate performance metrics to convey insights into why one prediction model appears to perform better than another. Data scientists need tools that would enable them to drill down to the context of each motor activity prediction to better interpret the causes of mispredictions. Existing tools using histograms of instance class probabilities are, however, insufficient for the visual analysis of activity classifications from sensor-data streams, as they do not consider the temporal context of predictions.

We introduce a novel visualization system, Track Xplorer (Xplorer for short), to interactively analyze the results of sensor-data classifiers. Xplorer enables users to debug and compare multiple classifiers down to the granularity of a single prediction (instance), providing qualitative and quantitative means to validate the performance of each model. Xplorer facilitates the interpretation of classification results in application context and thus lets data scientists reason about the causes of misclassifications and improve their predictive models.

Contributions. Our contributions include (1) a novel visualization system for visual analysis of classification performances on temporal data, (2) a set of methods to integrate contextual information and model metadata to support performance analysis, and (3) a visual track algebra for filtering, composing, analyzing and comparing classification results.
To illustrate the usefulness of our system, we present a use case in developing predictive models to detect specific motor activities in individuals with Parkinson's disease. We study the use of Xplorer through a group of fourteen participants, data scientists and business managers, working on the same project. We demonstrate that Xplorer proved essential in validating and comparing predictive models, reasoning about the causes of mispredictions, and understanding the trade-offs in the usage of different sensors-improving the overall predictive-model-development life cycle. We further observe how the system facilitates discussion among data scientists and business managers.

We first give a synopsis of prior work, followed by a brief discussion of our system design. We then provide details on Track Xplorer's interactions and visual design along with its track algebra, command-line set and classification validation support. Next we discuss the use of Track Xplorer in developing classifiers to detect movement patterns in Parkinson's disease. We conclude by summarizing our contributions and offering a take-home.

\section{Related Work}

Our work is related to earlier research in systems infrastructure for improving the machine-learning (ML) model development cycle, interactive analysis of classifier performances, sequential and temporal 
data query and visualization, and systems that facilitate visual analysis through algebraic operations.

\subsection{Systems Infrastructure for Improving ML Pipeline}

The practical success of ML in general and deep learning in particular has dramatically increased the demand for applying ML models to solve problems across domains. However, ML model development is iterative and time-consuming and often relies on trial and error using extensive engineering skills and large training data that are expensive and difficult to acquire and maintain [SHG* 15$]$.

Recent database research proposes systems to improve the MLmodeling life cycle. ModelDB [VSL*16] stores ML models along with associated pipelines and parameters, enabling the exploration of these models through SQL queries. Xplorer's visual track algebra specializes in comparative analysis of model predictions and performance and can be integrated into a SQL-like declarative language. ModelHub [MLDD17] provides a custom model-versioning system to track models and a domain-specific language to configure deep networks and their hyperparameters. Xplorer supports model tracking through integration with git, which is already used by developers to track the source code of their models. This avoids the burden and fragmentation of using two separate version control systems. Xplorer automatically extracts the metadata (e.g., parameters, training data info, etc.) for a given model along with its version from the model's source code in the git repository.

Training-data collection and management are also critical in end-to-end machine learning. Snorkel [RBER17] supports training-data generation at scale for ML models using weak supervision through user-defined labeling functions [RDSW* 16$]$. DataHub $\left[\mathrm{BCH}^{*} 15\right]$ supports managing datasets and their versions over time. Van der Weide et al. [vdWPS*17] tie data versioning to ML pipelines, tracking data resulting from intermediate stages of ML pipelines to reduce the redundancy of computations and improve their robustness. ProvDB [MCD17] tracks the provenance of artifacts (e.g., data, scripts, results) from data science experiments using git and a graph database. Similar to VisTrails [CFS*06], ModelDB, ProvDB, and [vdWPS* 17] also use workflows (pipelines) to represent modeling or analysis processes. However, unlike VisTrails, where users manually design the workflows, these three tools generate or infer workflows for users. Xplorer is akin to ProvDB [MCD17] in passively collecting model metadata. Unlike workflow-based systems, Xplorer focuses on a specific step in the ML pipeline: analysis of model prediction and performance. It is not intended to author and manage workflows for ML models. In this sense, Xplorer can be considered as a microservice that can be plugged into ML workflow management systems.

\subsection{Visual Analysis of Classifier Performance}

Researchers have introduced interactive tools, e.g., [RAL ${ }^{*} 17, \mathrm{ACD}^{*} 15$, $\left.\mathrm{AHH}^{*} 14\right]$, to help data scientists make sense of their classifiers' performances. Squares [RAL*17] supplements summary performance statistics with instance-level distribution information to uncover distinct characteristics of classifiers with comparable aggregate performance. Similarly, ModelTracker [ACD*15] and Confusion Wheel [AHH* 14] tightly couple performance with data instances to enable multiscale analysis.

These earlier tools combine histogram visualizations of instance class probabilities with aggregate metrics. However, the histogram visualizations of earlier work are not sufficient for drill-down analysis of sensor-data classifications, as they hide the temporal context of the classifier predictions. Track Xplorer lets users effectively inspect, compare, combine and reason about instance classifications while effectively visualizing the temporal context of these classifications. Xplorer also complements earlier work on classifier performance analysis by focusing on temporal data classifications, integrating additional "human soft knowledge" (e.g., activity videos and expert labels) and introducing a visual algebra over classification results that enables composable and rigorous performance analysis.

\subsection{Sequential Data Visualization and Querying}

The visual design of Xplorer is drawn from genomic data browsers (e.g. $\left.\left[\mathrm{KSF}^{*} 02, \mathrm{RTW}^{*} 11, \mathrm{SGM}^{*} 04, \mathrm{FWBB} 10\right]\right)$ and multimedia editors [App17, Ado17], using visual encoding along a linear axis (track) of data and metadata sequences as the basic unit of representation. Genomic browsers enable the visualization of molecular sequences from various sources as aligned linear tracks that can be added, removed and reordered on demand. Genomic browsers support interactions such as zooming and panning to enable fine-grained exploration of the data, often encoded as variable-length horizontal bars. These features are also common in multimedia editors, where tracks typically represent audio or video sources, and are shared by many other tools in the temporal and sequential data visualization literature [PMR* 96, WGGP* , HOB94, Kar94, BSM04].

To query temporal and sequential data effectively, prior research also proposes using a new temporal logic [Al183], SQL extensions [JS99, SAA*94], regular expressions [ZDFD15, CvW18], graphical languages [FKSSO6, MLMdO*13], and visual analogy with comic strips [JS09, JS10]. DecisionFlow [GS14] introduces a milestone-based analytics for event sequences, whereas EventPad [CvW18] applies a regular-expression-based visual language for identifying patterns of interest. Monroe et al.'s work [MLMdO*13] focuses instead on the specification of overlap and absence of interval events.

The primary use of our algebra is to generate new meaningful temporal sequences from the combination of existing ones through various operators, instead of matching event sequences of interest. Thus, our visual track algebra over temporal data-classification results complements earlier work on query-based selection.

\subsection{Visual Analysis and Design Through Algebraic Operations}

Earlier data visualization work proposes algebraic operators over data as well as visual encoding and design variables to formulate and compose complex hypotheses and visualizations. Polaris [STH02] introduces a table algebra drawn from Wilkinson's grammar of graphics [Wil05]. Vega-Lite [SMWH17] uses a composition algebra to construct layered and small-multiple views of visualizations. invis [DHHH13] provides an algebraic approach to inspecting RNA sequences represented as linear tracks, where mutations can be visually aggregated using the logical operators AND, OR, and NOT. Track Xplorer builds on earlier work and introduces an algebra that facilitates effectively manipulating, combining, and comparing track representations of classification results.

\section{Track Xplorer}

\subsection{Design Considerations}

Interacting with activity predictions based on sensor data poses a wide set of design challenges related to temporal and computational scalability. Running predictive models on large amounts of sensor data can take several hours and, depending on the sampling rate, can generate many predictions over time, making interactive analysis difficult. This is particularly noticeable when classifiers output continuous probability 


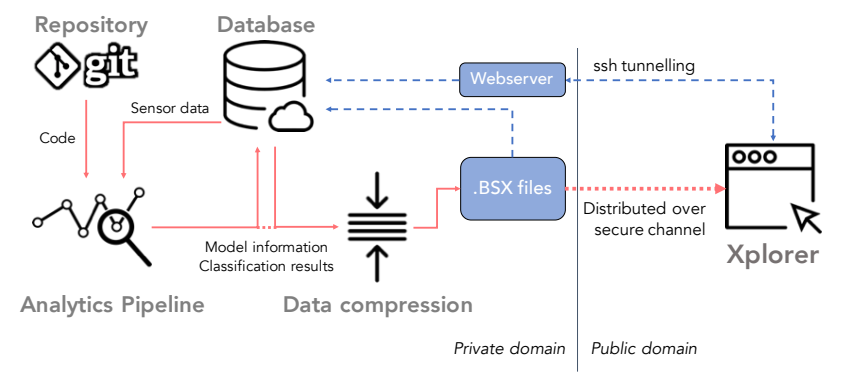

Figure 3: System design. Our analytics pipeline automatically loads models from the code repository and generates classification results for sensor data, storing the output in the database. Classification results and model metadata are then exported as a compressed (.BSX) file that Xplorer uses as input. Xplorer optionally supports direct access to the database through ssh tunneling (shown with blue arrows in the figure above), avoiding the need for sharing .BSX files.

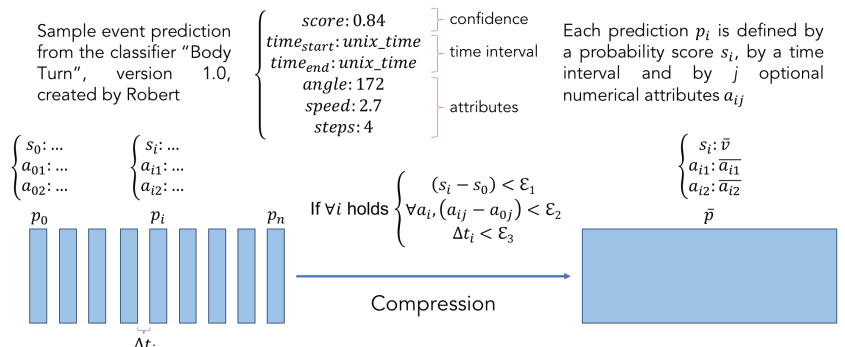

Figure 4: Data compression. Prediction results adjacent to each other in time and meeting specific similarity criteria can be aggregated into a single longer prediction. For each classifier prediction $p_{i}, s_{i}$ denotes its confidence value, $a_{i j}$ its attribute values and $\Delta t$ its time distance from the end of the previous prediction. Compression is performed if these values do not vary beyond certain thresholds $\varepsilon_{k}$ from the first prediction.

values over time through a sliding-window approach, which can generate multiple prediction samples per second.

Our system tackles these issues through automated precomputation and data compression. In particular, we rely on a set of procedures that periodically handle accessing the available predictive models, running them on the right subset of the sensor data, and compressing and storing their output (Fig. 3). Data scientists are required to include in their code a standardized set of attributes that hold meta-information about the nature, version and requirements of a classifier. This information is complemented by the git version control system, which holds data about user commits and modifications in the code. An analytics pipeline checks the code repository for new versions of the models and runs them when a change is detected, performing the computation according to the meta-information extracted from each classifier. Results are then stored in a centralized database together with the classification results. By merging similar predictions close to each other in time (Fig. 4), we compress the results and store them in a JSON-based file (.BSX) that can later be opened from the public, web-based Xplorer user interface.

An important design criterion for our system involves preserving data confidentiality while allowing easy distribution of classification results. Since data science projects are often run across groups or companies,

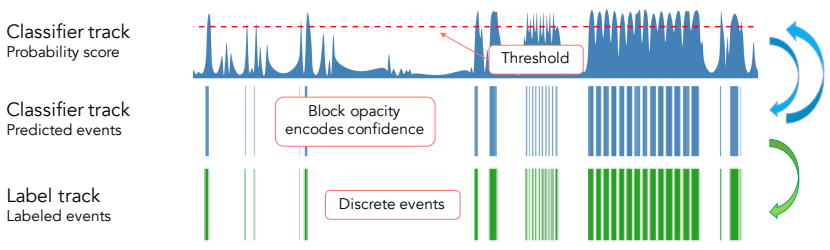

Figure 5: Track definition. A track in Xplorer corresponds to a list of non-overlapping time periods ("events"). The two track types are based on the form of the data they represent: classifier tracks and label tracks. A classifier track contains probability scores associated to each event and can be visualized either as an area chart or as horizontal bars ("blocks"), whereas a label track contains only information about time intervals. A classifier track can be converted into a label track by applying a threshold on the classifier track's prediction scores.

it is common to maintain separate access privileges for prediction results, sensor data and source code. Distributing BSX files means that any user can easily be given access to prediction results through the publicly available Xplorer interface, without being exposed to any confidential information. On the other hand, data scientists can easily enable an optional, protected ssh tunneling connection (Fig. 3) to access confidential information without having to deal with BSX files.

While we here propose a very specific system architecture to optimize the overall model development cycle, Xplorer can also be used as a standalone tool to handle the import of classifier predictions, ground-truth labels or generic time intervals from standard CSV files.

\subsection{User Interface}

The interface of Xplorer (Fig. 2) is composed of a main view in which classification results and labels are represented as linear tracks stacked vertically. A track visually corresponds to a sequence of non-overlapping colored blocks positioned over a common timeline. We categorize tracks into two types based on the form of the data they visualize: classifier tracks and label tracks (Fig. 5). Although here we represent classifier tracks in blue and label tracks in green, we note that both the color of each track and their vertical arrangement are freely customized by the user.

In classifier tracks (Fig. 2a), a block corresponds to a single prediction or to a set of consecutive identical predictions, and this may result in blocks of variable length. If the output of a classifier is binary, the block is visible only when the activity is detected. If a classifier outputs continuous probability scores, the block is generated by applying a classifier-specific threshold to the score values (Fig. 5). The opacity of a block encodes the associated probability score, increasing with high score values and decreasing with low score values. When dealing with multi-class classifiers, Xplorer generates by default a separate track for each predicted class, leaving an option to aggregate them visually.

In label tracks (Fig. 2b), each block corresponds to a textual label (e.g. "Walking," "Person is sitting") characterized by a start and end time that determine its position and length. Labels can be either algorithmically generated or manually defined by a human, and are often used as groundtruth or as a reference for validating classifier tracks. A particular type of label track, called protocol track (Fig. 2c), can hold different unique labels on the same timeline, given they do not overlap with each other. A protocol track is generally used as a reference to the data collection process, where each block corresponds to a specific task performed by a subject. 


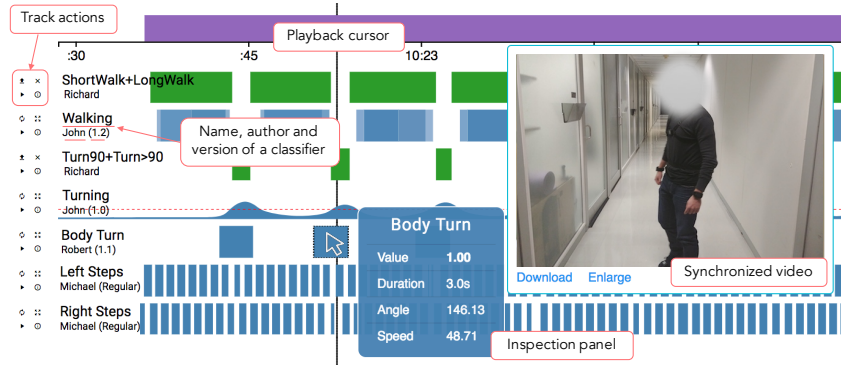

Figure 6: Sample track configuration in Xplorer. It is obvious from the pattern of green and blue boxes (label and classifier tracks, respectively) that the subject is alternating between walking and turning movements. Visual alignment, video playback and variable inspection can be used in combination to better understand predicted motor events.

While all other temporal data is represented in linear tracks, video is shown in a separate undocked window (Fig. 2d), which can be dragged across the interface and freely resized by the user. The interface of Xplorer also includes a left sidebar from which users can decide which tracks to visualize and can easily zoom to specific events in the protocol track, and three auxiliary modal windows. These latter windows can be used for (1) analyzing classifier performance, (2) inspecting model information and code revisions, and (3) accessing raw sensor data associated to a particular prediction or time interval.

\subsection{Interactions}

Track information can be analyzed at different levels of granularity through zooming and panning, which are performed with the mouse wheel and drag actions. Hovering on a block displays information about the correspondent prediction or label (e.g. author, duration) as a tooltip. For classifier tracks, the tooltip shows classifier-specific variables associated to the prediction (e.g. "tremor frequency," "angular velocity") that data scientists can use to debug their algorithms (Fig. 6).

Each classifier track also includes four buttons enabling the user to (1) increase its height for better visibility, (2) play consecutively the videos of all detected activities, (3) display information about the underlying predictive model (e.g. sensors, prediction window and threshold used), and (4) switch between two different visualization modes (area chart or horizontal bars). Fig. 5 explains how a classifier track can also be represented as an area chart, visualizing a continuous probability score over time. This mode is particularly useful for observing how a classifier's threshold determines which events are detected (and thus generate a block). The threshold can be dynamically changed by the user by moving the red horizontal line shown in Fig. 5, thus avoiding the recomputation of classification results. All tracks can be vertically rearranged by dragging, so that users can better compare them by placing tracks of interest next to each other.

\section{Visual Track Algebra}

While analyzing each track separately may be sufficient for some applications, in many cases the ability to combine different tracks can be essential. For instance, a user may want to analyze the output of a tremor classifier only when a different classifier predicts no walking movement. Similarly, a user wanting to consider all moments in which a subject is stationary needs to unify the labels associated to "Sitting" with labels associated to "Standing". To enable reasoning beyond the

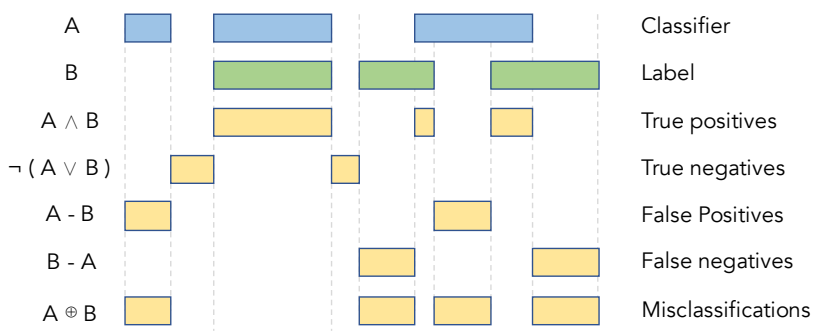

Figure 7: Validation of classifiers through track algebra. A represents a classifier track and B represents a label track containing ground-truth labels. By computing the intersection and subtraction of the two tracks, users can quickly identify correct and incorrect predictions.

scope of single classifiers and labels, we define a visual algebra that can generate new tracks as combinations of existing tracks. Operations such as addition, subtraction, logic conjunction and disjunction can be applied to both classifier and label tracks with different semantic meaning.

Fig. 7 illustrates how the most common operators can be used for classifier validation. If we denote a classifier track by $A$ and a label track used as ground truth by $B, A \wedge B$ corresponds to the intersection of both tracks, that is to the events that were correctly predicted by the model (true positives). Similarly, we can define the difference between track $A$ and track $B$ as a new track composed of instances of $A$ not present in $B$. This way, the track $A-B$ contains all classifications that do not match any ground-truth label (false positives), while $B-A$ conversely represents labeled events not identified by the predictive model (false negatives).

The power of the track algebra lies in enabling users quickly to combine tracks to validate complex hypotheses about the classification process. In particular, in the presence of ground-truth labels, identifying misclassified events is visually straightforward. In combination with the video functionality, the track algebra also enables the consecutive playing of all false positive and false negative predictions for a particular classifier. This way, the user can visually validate the performance of his predictive model and reason about the causes of each single misprediction based on its context.

\subsection{Command Line: Combining, Filtering and Ordering}

Track Xplorer interface features a command-line interface that lets users quickly perform complex interactions, such as track manipulation through visual algebra. Fig. 8 lists the most common commands executable from the command line. Each command is composed of one operator and one or two operands, which can be track identifiers or numerical values. A track identifier is automatically generated as a combination of the track name, author and version (e.g., the first version of the "Sleeping" classifier created by author "John" generates the ID "SleepingJohn1.0") and is made available by auto-completion. For instance, when "threshold sleep" is typed, the command line automatically infers which available track is best suited to the operator "threshold", highlights it in the main view, and offers the suggested completion "threshold SleepingJohn1.0". Tracks can also be referred by their order of appearance (e.g. "union 12 " generates the union of the first two tracks) or by string wildcards (e.g. "show \%walk" makes all tracks related to walk visible). When a command generates a new track, it is added to the main view and its name and identifier are defined automatically from the operation performed. The command line can also be used to compute performance metrics, order tracks, and filter classification events based on their attributes. For instance, the 
Marco Cavallo \& Çăgatay Demiralp / Track Xplorer

\begin{tabular}{rccc}
\hline Operator & $P_{1}$ & $P_{2}$ & Description \\
\hline negate & $T$ & & Generates $\neg T$ \\
add / union & $T_{1}$ & $T_{2}$ & Generates $T_{1} \vee T_{2}$ \\
intersection & $T_{1}$ & $T_{2}$ & Generates $T_{1} \wedge T_{2}$ \\
errors & $T_{1}$ & $T_{2}$ & Generates $T_{1} \oplus T_{2}$ \\
subtract & $T_{1}$ & $T_{2}$ & Generates $T_{1}-T_{2}$ \\
match & $T_{1}$ & $T_{2}$ & Generates $T_{1} \sim T_{2}$ \\
variation & $T_{1}$ & {$\left[T_{2}\right]$} & Generates $T_{1} \partial T_{2}$ \\
play & $T$ & & Plays all events in track $T$ \\
threshold & $C$ & Float & Changes C's threshold to a fixed value \\
show / hide & $T$ & & Shows / hides track T \\
transform & $C$ & & Thresholds $C$ and generates an $L$ track \\
rename & $T$ & & Renames a track \\
color & $T$ & Color & Changes the color of track T \\
author & $A$ & & Shows author A's tracks \\
filter & $T$ & Exp & Selects events of T that satisfy Exp \\
order & & & Smart ordering based on current time \\
info & $T$ & & Display detailed information on a track \\
jaccard & $T_{1}$ & $T_{2}$ & Jaccard distance between $T_{1}$ and $T_{2}$ \\
roc & $C$ & $L$ & Computes ROC curve and AUC score \\
report & $C$ & $L$ & Computes precision, recall and F1 score \\
score & $T$ & & Computes the match score metric \\
\hline
\end{tabular}

Figure 8: Commands of the Xplorer command line. $P_{1}$ and $P_{2}$ are the parameters required by each command. $T$ is a placeholder for a generic track's identifier, whereas $C$ and $L$ indicate a classifier and a label track respectively. Track type conversion is handled automatically according to Fig. 5.
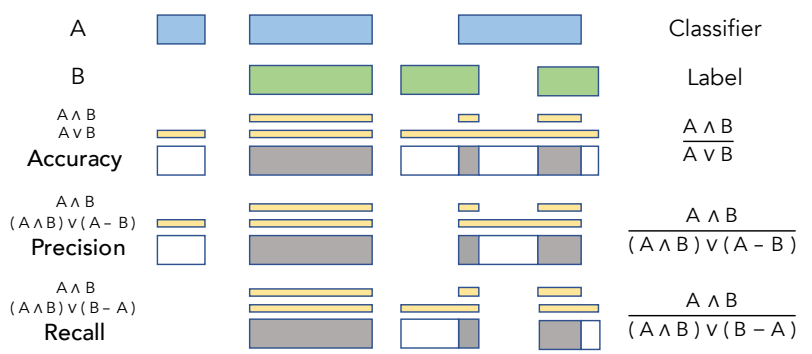

Figure 9: Common performance metrics computed through track algebra. $A$ is a classifier track and B is a label track containing ground-truth labels. Accuracy, precision and recall scores can be visualized as a "container" track with partial color fill. Mispredictions affecting a specific performance metric are localized in the non-filled (blank) regions of the track.

command "filter TurningErhan 1.2 angle $>60$ \&duration $>2$ " creates a new track containing only slow-turn events that last more than two seconds and in which the subject rotates by more than sixty degrees. When a moment $t$ along the timeline is chosen with the cursor, the command "order" instead selects all tracks containing an event overlapping the time interval $(t-\varepsilon, t+\varepsilon)$, and orders them based on their temporal match.

\subsection{Classifier Validation}

When a classifier track $A$ and a ground-truth label track $B$ are observed next to each other, it is intuitively clear that the performance of the predictive model depends on how extensively the blocks of each track are aligned with each other. Optimally, for each block in $A$ there should exist a block in $B$ of equal length whose start and end points match those

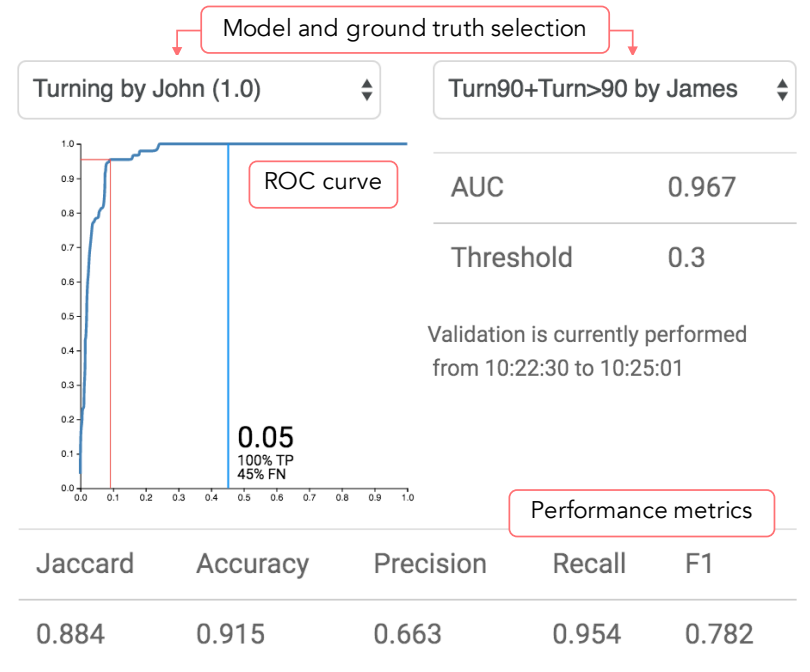

Classifier Information: Walking

\begin{tabular}{|c|c|c|c|}
\hline & Model & \multicolumn{2}{|c|}{ Walking version 1.1, created by John } \\
\hline 200 & Contributors & Robert ( 2 commits) & Model metadata \\
\hline$\cong$ & Dependencies & \multicolumn{2}{|c|}{ Opals: Sternum (accelerometer, gyroscope) } \\
\hline c & Specifications & \multicolumn{2}{|c|}{ Frequency: $10 \mathrm{~Hz}$, Time window: $2 \mathrm{~s}$, Threshold: 0 . } \\
\hline (\$) & Notes & \multicolumn{2}{|c|}{ Recurrent Network, LSTM-based } \\
\hline\langle\rangle & Development & \multicolumn{2}{|c|}{ Oct 17 - Nov 6 ( 19 days). 6 commits in total } \\
\hline \multicolumn{4}{|c|}{$\begin{array}{l}\text { Nov 6, d2c38db by John: Revert layer to old params } \\
\quad \text { Istm.py }\end{array}$} \\
\hline$x$ & recurrent_layer.py & & Latest commits \\
\hline
\end{tabular}

Figure 10: Performance metrics (top) and classifier information (bottom) modal windows. Xplorer features a modal window (top) to display different performance measures for a classifier track and includes an interactive ROC (Receiver Operating Characteristic) curve to help the user choose an adequate threshold for the selected model. The information modal (bottom) displays model metadata and summarizes commits and modifications performed on the code repository.

of $A$. However, misclassifications and other prediction-related errors may misalign or eliminate one of these two blocks.

A straightforward numerical way to quantify the visual overlap of two tracks is the Jaccard index, defined as the intersection over union of two series of values. If we define the "length" of a track as the sum of the duration of all its blocks, the Jaccard index simply corresponds to the length of the intersection of two tracks $(A \wedge B)$ divided by the length of their union $(A \vee B)$.

Leveraging the definitions in Fig. 7 lets us extend this example and express common performance metrics such as accuracy, precision and recall scores in terms of visual track algebra (Fig. 9). In particular, we can define a new type of track in which a set of "empty" boxes is defined by the denominator of the formulas in Fig. 9 and is filled according to the numerator of the fraction. Non-filled regions of the track correspond to mispredictions 
affecting a specific performance metric, whereas filled regions correspond to the contributing correct predictions. While performance metrics are generally expressed as aggregate numerical values, our method also visualizes them spatially - enabling data scientists to understand how these proportions are computed, to identify where mispredictions are located in time, and to discern which of them affect a specific metric of interest. For instance, the diagram in Fig. 9 makes it clear that the value of the recall performance metric is unaffected by the first misprediction of classifier A.

Since performance metrics often depend on the threshold applied to the continuous prediction of a classifier, we include in the Xplorer interface a modal window displaying an interactive Receiver Operating Characteristic (ROC) plot (Fig. 10) with its related Area Under the Curve (AUC) score - a threshold-independent performance metric. By dynamically changing the threshold, the user can balance the number of true positives and false negatives and observe how tracks and metrics are updated.

\subsection{Advanced Algebraic Combinations}

So far we have primarily described how to apply our visual track algebra between a classifier track and label track containing ground-truth labels. In real-life scenarios, the development of predictive models can benefit from applying algebra on a wide variety of track combinations, even over more than two tracks at a time.

It can be meaningful to apply our algebra over two classifier tracks. Computing the intersection of two classifiers (Fig. 11a) shows how greatly their event detections correlate. Alternatively, one can combine multiple classifiers to estimate how they would perform if executed together. As an example, if a predictive model has many false positives because the subject is performing a different activity, a second classifier that detects that activity and removes the outliers (Fig. 11b) could be useful.

Often the generation of ground-truth information for motor activities entails multiple people manually annotating the same dataset. Since motor activities are perceived slightly differently by annotators, evaluating performance based on their agreement (Fig. 11e) is often useful. Track algebra can also be used to exclude from validation all cases in which ground-truth labels (Fig. 11d) could not be collected, thus guaranteeing a more legitimate performance estimate. Similarly, mutually exclusive labels can be combined to exclude unclear motor events (Fig. 11f).

Finally, we note that classification performance in activity detection is highly affected by how much predictions and labels overlap over time. Sometimes it is of interest to precisely matching the start and end time of each motor event, whereas in other instances event detection is sufficient. In the latter case, precise overlap of two tracks is not a reasonable performance metric. We thus introduce the " $~ "$ operator, a variation of the subtraction operator that is not sensitive to the start and end of each detection (Fig. 11c). Applications of the advanced algebraic operations introduced here are discussed further in the next section.

\section{Use Case: Detecting Motor Activities in Parkinson's Subjects}

We present here the application of our system to a project involving predictive models to automatically detect specific motor activities by subjects affected by Parkinson's disease. Xplorer was used as a companion tool over most of the project by a mixed team of fourteen data scientists, domains experts and business people.

Six wearable IMU sensors were worn by 25 Parkinson's disease subjects over multiple sessions (visits) of about an hour's duration. The sensors measured accelerometer, gyroscope and magnetometer information at
$128 \mathrm{~Hz}$ and were placed on the wrists, feet, chest and back of the patients. During each visit, all subjects performed the same set of predefined tasks according to a single clinical protocol. Technicians not involved in the system development work handled recording sessions, labeling specific activities and time-stamping the task executions. Sensor data, ground-truth labels and video files were all stored in a single database that all data scientists could access as they developed their predictive models.

Data scientists developed their algorithms in Python, in accordance with the specifications of our analytics pipeline. Their code was pushed to a private GitHub repository and automatically loaded and executed by our system. The results of the computation were systematically made available to the team through the Xplorer user interface, with no additional input from the group members. Classification results were analyzed both individually and collaboratively during weekly team meetings.

\subsection{Classifier Development and Comparison}

Developing a predictive model is an iterative, trial-and-error process made up of analyzing a first set of classification results, tuning model parameters and then recomputing predictions. Xplorer proved valuable to data scientists in discovering insights as they analyzed classification results.

Video Playback and Track Algebra. The playback functionality, in combination with the track algebra, proved to be fundamental in quickly identifying mispredictions. For example, subtracting the "Walk" label track from the "Walk" classifier track and playing the resulting track showed all cases in which the classifier did not detect that the subject was walking (false negatives). By observing the video and the task labels, data scientists realized that, since the model was using a sensor worn on the chest, it was incorrectly classifying movements such as rising from the chair and buttoning a coat (Fig. 12a). Similarly, the "Step detector" classifier track (based on shoe-worn sensors) showed false positives corresponding to foot tremor (Fig. 12b), particularly common when subjects were sitting with their legs crossed. Exploiting these insights, data scientists decided to retrain their classification models, either by using data from different sensors or by including in the training set the activities that had been misclassified.

Model Debugging. Another widely used feature was the ability to inspect information about each single prediction. After noticing that the two hand classifiers "Pronation-supination" and "Tremor" were biased by the action of walking, data scientists were able to mouse over mispredicted events and observe the attributes computed by their predictive models (Fig. 12d). In this case, each prediction held numerical information about hand rotation angle, hand rotation speed, tremor frequency and tremor amplitude. By analyzing these attributes, data scientists were able to filter out movements happening at specific frequencies associated with walking, thus making their model more robust. Similarly, the filtering function was used to check the validity of step detections and revealed several events characterized by unexpectedly long durations and unrealistic speeds (Fig. 12b). Isolating these events and playing their associated video showed that the stepdetection algorithm did not properly detect the foot-landing phase.

Classifier Versioning. A key feature of Xplorer is the ability to compare the results not only of different classifiers, but also of different versions of the same predictive model. Not always updating a model may yield better performance, and tracking changes is important in understanding which modifications have led to an improvement or should be reverted. Version comparison was typically used to tune model parameters to balance the number of false positives and false negatives, in particular for classifiers such as "Sit2Stand" and "Tremor" (Fig. 12d). 


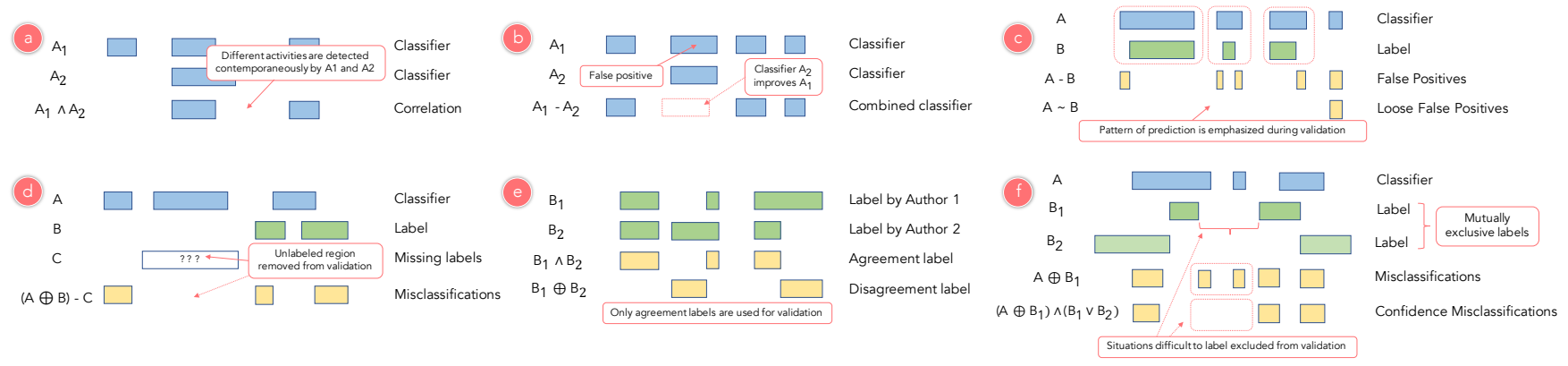

Figure 11: Advanced algebraic applications. Track algebra can also be applied among classifier tracks, e.g., to quantify their correlation (a) or to estimate their combined performance if they were executed together $(b)$. Other relevant applications relate to obtaining a more legitimate performance estimate when dealing with missing labels $(d)$, unclear motor events $(f)$, or multiple sources of ground-truth information (e). The “ ” operator is also introduced to emphasize event detection over precise temporal matching (c).
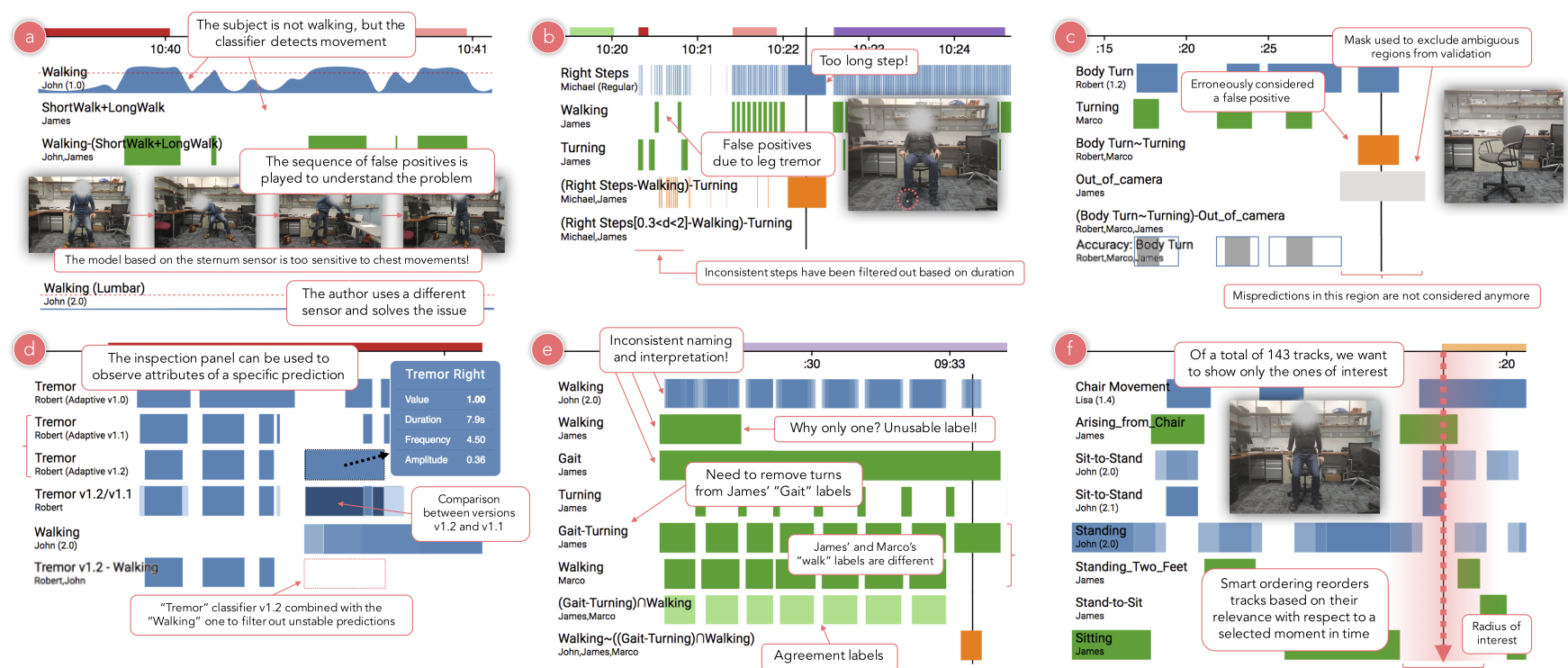

Figure 12: Sample screens from our use case: (a) combining track algebra and video playback to identify false positives; (b) using filtering to identify and remove tremor mispredictions; (c) removing regions of uncertainty from validation; $(d)$ comparing classifier version and inspecting attributes; (e) handling inconsistent labeling with track algebra; $(f)$ using smart ordering to identify tracks of interest.

\subsection{Label Accuracy and Validation}

While human-generated labels may seem a valid source of ground-truth information, blindly assuming their completeness and correctness can easily lead to wrong insights and inaccurate performance estimates. A mislabeled time period, for instance, can erroneously reveal a false-positive or false-negative prediction, thus decreasing the classifier's performance and creating a bias in interpreting its classification results.

Partial Labeling. The first issue encountered in this project was the discrepancy between the ground-truth labels provided by video annotators (who were instructed using a pre-existing medical protocol) and those requested or expected by data scientists (who were more aware of machinelearning requirements for training). In many cases, such problems could be easily solved by combining labels through track algebra. For instance, while raters defined partial labels such as "ShortWalk" and "LongWalk" or "Turn90" and "Turn $>90$ ", data scientists could combine them in single tracks ("ShortWalk+LongWalk", "Turn90+Turn>90") and then use them in evaluating the performance of their classifiers (Fig. 6).

Missing Labels. Another problem involving manual video annotations was a sporadic absence of labels, either due to distraction of the technician or to the subject being off camera. In evaluating the performance of the "Walking" classifier through track algebra, the team found a percentage of false positives much higher than expected. Playing the corresponding parts of the video revealed that the subject had walked outside the camera view, where the video annotator couldn't tell what actions were performed. Here, the annotator was asked to generate a new label track indicating all moments in which the patient was off camera, and these were then excluded from the performance evaluation via track algebraic subtraction (Fig. 12c).

Label Definition and Human Bias. In looking more minutely at mispredictions, the team observed that among the causes of lower performance was the definition of motor activities themselves. For instance, 
while the "Walking" classifier was trained to recognize any horizontal movement involving foot motion, the annotator's definition of the motor activity required a minimum of three steps by the patient in the same direction. Because of this, the track algebraic difference showed a large set of false positives associated to small movements, such as performing few steps in reaching an object. Similarly, an inconsistent definition of "turn" was used for generating labels. Typical questions in team meetings included "Should a larger rotation of the chest be considered a turn, even if the legs don't move?", "Which is the minimum angle of rotation that defines a turn?", and "To what extent it is useful to consider such details for the purpose of the project?'. Fig 12e shows how these inconsistencies in labeling were handled through track algebra. Visually matching the alignment of predictions revealed human bias in the annotation of motor activities. For instance, it was discovered that some "walking" labels were assigned before the patient actually started moving his feet. This mislabeling probably occurred because the video annotator already expected the intention to move of the subject, whose actions had to follow a standard medical protocol. During performance evaluation, data scientists could limit these difficulties by considering detection patterns through the " $\sim$ " operator (Fig 11c). To mitigate this problem, the data scientist team asked the technicians to provide multiple ground-truth labels by different video annotators for a given activity. Thanks to Xplorer's track intersection functionality (Fig. 11e), data scientists could then run performance evaluations only in regions where the annotators agreed (Fig. 12e).

\subsection{Team Collaboration}

While individual data scientists used Xplorer for classifier development, the visualization tool was also used collectively by the entire team during weekly meetings. In particular, its visual output was projected on a large screen or shared with remote participants via video conferencing. Every week, the tool would be used to show progress on acquiring new patient data, developing classifiers and generating new ground-truth labels. The person acting as moderator also used Xplorer to review team progress and lead the conversation.

Collaborative Interpretation. Due to its abstraction and simple visuals, Xplorer proved to be an efficient medium for discussion among people with very different backgrounds. Lack of familiarity with machine learning or with the details of each predicted model was no impediment for team members in visually checking the alignment of tracks and further validating them with the video. Each data scientist could observe and give feedback about models built by other developers without knowing all their implementation details. Domain experts as well were able to share their knowledge of Parkinson's disease and advise data scientists how best to handle certain motor-activity events. Similarly, data scientists could express through Xplorer the need for additional training samples or higher- quality ground-truth labels.

Decision Making. Xplorer was used managerially to track the progress of the project with respect to deadlines and to assess the quality of each predictive model before deployment. Human resources were dynamically allocated in order to compensate for predictive models that showed weaknesses in Xplorer. For instance, using the tool quickly led to a discussion of the quality and reliability of labels and suggested that management consider hiring new personnel to annotate videos manually. Similarly, false negatives shown in Xplorer were used to justify the acquisition of new data from additional patients in order to have enough samples for each Parkinson's phenotype. Consideration of classifier accuracy from the video footage led to the exclusion of a subset of the sensors from the project scope, a decision that led data scientists to focus more on wrist and lumbar sensors.

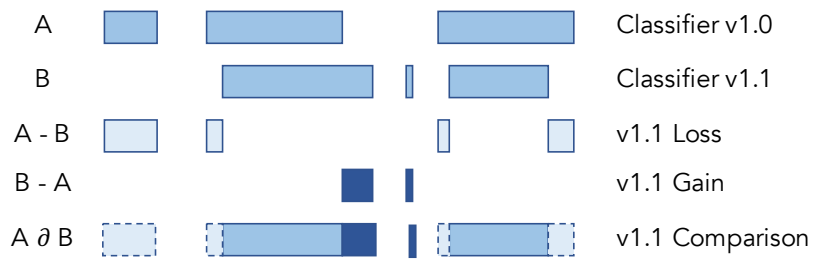

Figure 13: Classifier versioning. Track Xplorer supports a hybrid versioning based on the combination of classic version control systems (e.g. git) and a standardized model definition. Track algebra can also be used to visualize differences between classifier versions.

\section{Discussion}

\subsection{Improving Scalability at Multiple Levels}

Users can exploit five main data components in performing visual analysis of classification results: (1) the actual results of the analysis, (2) ground-truth labels, (3) multimedia footage, (4) raw sensor data and (5) additional metadata information. While (3) can be consumed via streaming and (5) generally uses only a limited amount of memory, the number of instances of (1), (2) and (3) can cause serious scalability issues that affect the interactivity of the system. We resolve these issues by reducing the frequency of (1) and (2) events through the compression algorithm in Fig. 4, and by dynamically loading (3) only for small subsets of the dataset, based on user request.

A different scalability limitation lies instead in the number of tracks visualized on screen, which amounted to almost 100 in our use case scenario. Too many classifier or label tracks displayed at the same time can make visual search and comparison cumbersome for the user, who would prefer a small, ordered set of tracks so as to achieve the desired visual insights. For this reason, we combine the use of smart autocompletion and wildcard selectors in the command line with a smart ordering functionality.

Smart track ordering addresses the situation in which the user is interested in a particular moment in time and wants to visually compare all tracks containing relevant information in that particular time window. Say, for instance, that the user is focusing on the moment in which the subject is standing up from a chair and wants to see tracks that detect events related to this action. Smart ordering automatically reorders classifier and label tracks, bringing Arising from chair, Sit-to-Stand, Sitting and Standing close to each other. Smart ordering further remembers previous tracks of interest, so that if the user focuses on a consecutive action in which the user sits back on the chair, the track Stand-to-sit is ordered on top and the track Sit-to-stand (no longer involved) remains visible.

\subsection{A First Step Towards Model Versioning}

As machine learning technology matures, research has focused more and more on creating systems to build models more effectively. However, despite the fast expansion of machine-learning applications into new domains, many aspects of the predictive modeling life cycle have not yet been properly addressed. Track Xplorer relies on enforcing a standardized model definition that all data scientists commit to use, but leaves them free to continue using git as a versioning system. In particular, Xplorer's analytics pipeline automatically extracts model metadata information from git commits and presents it in the front-end user interface (Fig. 10, bottom) while comparing multiple versions of the same classifier. Data scientists can use the track algebra to identify differences between model 
Marco Cavallo \& Çağatay Demiralp / Track Xplorer

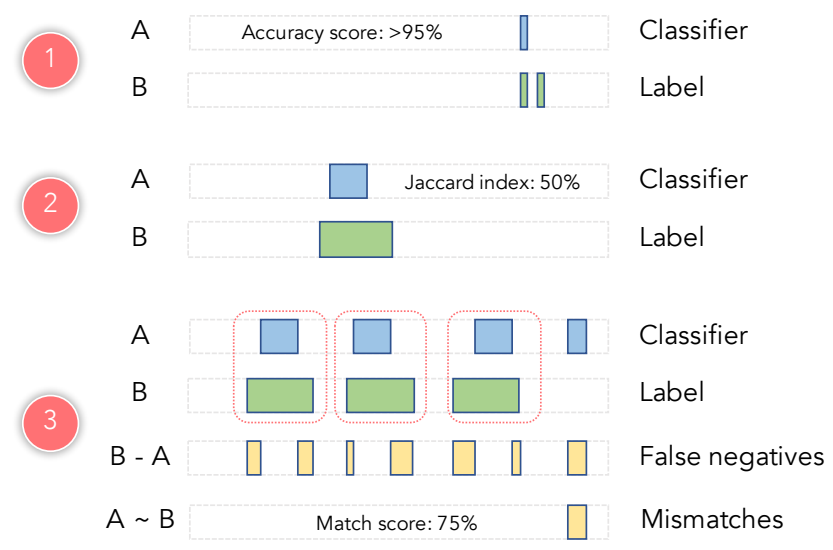

Figure 14: Enhanced performance analysis through visual track algebra. Track Xplorer lets data scientists reason about the tradeoffs of each performance metric, helping them decide which ones to adopt. It also helps them gain insights for developing new, effective performance metrics tailored to the classification task at hand.

versions (Fig. 13) and then inspect which parameters and commits are associated to the best results. This way, data scientists can easily go back to their code and revert or confirm modifications to their model.

\subsection{Running Meaningful Performance Evaluations}

As shown in Fig. 9, our track algebra also supports visualizing over time the contributions of predictions to the computation of common performance metrics. Key in evaluating the performance of a predictive model is choosing which quality metrics to adopt — and the decision is often based on the desired outcome of the prediction. For instance, let's suppose we are interested in detecting the event of a subject standing from a seated position, but we are not concerned with detecting a precise time window for this movement. In case (1) of Fig. 14, we would correctly predict one Sit-to-Stand event out of two, after a long period in which no events are detected. If we consider the fact that the classifier correctly predicted that no other events happened over that period, the accuracy metric would tell us that our model is performing extremely well (beyond 95\% correct predictions over time). However, for our purposes, this metric is extremely misleading, since the classifier missed half of the interesting events. Similarly, the Jaccard index computed in case (2) of Fig. 14 would suggest a $50 \%$ overlap, but this is not very meaningful if we are interested in event detection only. The simple concepts of false positives and false negatives in fact assume subjective meanings in time-series data classifications. Fig. 14(3) shows how the detection of four events would by definition generate seven sequences of mispredictions, with a very negative impact on most performance metrics. Xplorer enables data scientists to visually assess the suitability of a particular metric, but also encourages the definition of new performance estimators. For instance, in Fig. 14(3) we could approximate the overall performance by applying the match operator of our track algebra, which identifies only one mispredicted event. Visually, this leads us to define a new and more suitable empirical performance metric that simply corresponds to the number of correctly detected events (in this case, three out of four, i.e. $75 \%$ ).

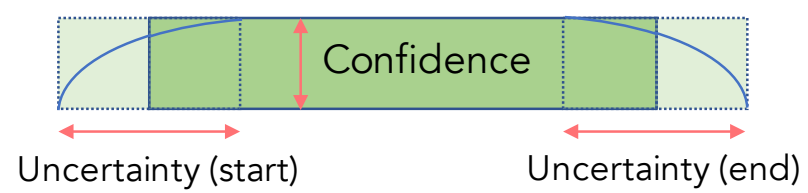

Figure 15: A probabilistic approach to ground truth information. Labels could be better modeled as a function over time to take into account the confidence on the event and the uncertainty in precisely identifying its start and end timepoints.

\subsection{A New Model for Activity Labeling}

Another issue affecting performance evaluation is the quality of groundtruth labels, which we treated simply as actions defined through a start and end moment in time. As discussed in our use case, video annotators cannot always find precise time boundaries for a motor activity and at times are not even sure if an action should be labeled at all-with predictably affecting the subsequent validation process. It may be useful instead to approach ground-truth labels probabilistically, similarly to what is currently done for classifiers. For instance, a ground-truth label could be defined as a function whose value depends on the confidence of the video annotator, with time boundaries modeled so as to take into account the uncertainty in determining the exact moment at which the action started or ended (Fig. 15).This would make it possible to devise new performance metrics and define a continuous version of our current track algebra, with broader possibilities for model analysis and manipulation.

\section{Conclusion}

We introduce Track Xplorer, a system for interactive visual analysis of predictions of classifiers modeled to detect events in temporal sensor data. Our system enables the user to visually and quantitatively analyze and compare results from multiple classification models, improving the model development and debugging experience of data scientists. Track Xplorer couples contextual information such as ground-truth labels, expert annotations and event videos together with track visualizations of predictions through interaction and visual encoding, thereby empowering users of diverse backgrounds to better interpret, debug, and enhance classifier performance. We also introduce an extensible visual algebra over track representations that supports composable and rigorous performance comparison and analysis.

We demonstrate the usefulness of our tool through its application in a collaborative project for classifying motor activity patterns to score the degree of disease progression among Parkinson's disease patients. Track Xplorer enables the project team members to identify early on possible systemic errors in the data, reason about and find the causes of misclassifications, and effectively compare the results of different classifiers, thus improving classification performance by selecting better models and parameters.

Research into systems for machine learning model development and management is nascent but burgeoning. These systems would benefit from integrating data visualization and visual analytics as a first-class citizen for improved user experience. Track Xplorer is the first system that enables visual and quantitative analysis, comparison and tracking of temporal multisensor data classifications across models.

\section{Acknowledgments}

We thank the BlueSky project members at IBM Research for their feedback on Track Xplorer and earlier drafts of this paper. 


\section{References}

[ACD*15] Amershi S., Chickering M., DruCKer S. M., LeE B., SIMARD P., SUH J.: ModelTracker: Redesigning performance analysis tools for machine learning. In Proc. CHI (2015), ACM, pp. 337-346. 1, 3

[Ado17] Adobe Corporation: Premiere Pro. http://www.adobe. com/products/premiere. html, 2017. Accessed: 2017-07-31. 3

[AHH*14] Alsallakh B., Hanbury A., Hauser H., Miksch S., RAUBER A.: Visual methods for analyzing probabilistic classification data. IEEE TVCG 20, 12 (2014), 1703-1712. 1, 3

[All83] ALLEN J. F.: Maintaining knowledge about temporal intervals. Commun. ACM 26, 11 (1983), 832-843. 3

[App17] APPlE CORPORATION: iMovie. https://www.apple.com/ imovie/, 2017. Accessed: 2017-07-31. 3

[BCH* 15] Bhattacherjee S., Chavan A., Huang S., Deshrande A., Parameswaran A.: Principles of dataset versioning: Exploring the recreation/storage tradeoff. Proceedings of the VLDB Endowment 8, 12 (2015), 1346-1357. 3

[BSM04] BADE R., SCHLECHTwEG S., MiKsCH S.: Connecting time-oriented data and information to a coherent interactive visualization. In Procs. $\mathrm{CHI}$ (2004), ACM, pp. 105-112. 3

[CFS*06] Callahan S. P., Freire J., Santos E., Scheidegger C. E., Silva C. T., Vo H. T.: Vistrails: visualization meets data management. In Proceedings of the 2006 ACM SIGMOD international conference on Management of data (2006), ACM, pp. 745-747. 3

[CvW18] CAPPERS B. C., VAN WIJK J. J.: Exploring multivariate event sequences using rules, aggregations, and selections. IEEE TVCG (Proc. InfoVis) (2018). 3

[DHHH13] Demiralp C., Hayden E., Hammerbacher J., HeER J.: invis: Exploring high-dimensional rna sequences from in vitro selection. In Proc. BioVis (2013), IEEE, pp. 1-8. 3

[FKSS06] FaIls J., Karlson A., Shahamat L., ShNeIderman B.: A visual interface for multivariate temporal data: Finding patterns of events across multiple histories. In Procs. VAST (2006), pp. 167-174. 3

[FWBB10] Fiume M., Williams V., Brook A., Brudno M.: Savant: genome browser for high-throughput sequencing data. Bioinformatics 26, 16 (2010), 1938-1944. 3

[GS14] Gotz D., Stavropoulos H.: Decisionflow: Visual analytics for high-dimensional temporal event sequence data. IEEE transactions on visualization and computer graphics 20, 12 (2014), 1783-1792. 3

[HOB94] HARRISON B. L., Owen R., BAECKER R. M.: Timelines: An interactive system for the collection and visualization of temporal data. In Procs. Graphics Interface (1994), pp. 141-148. 3

[JS99] JENSEN C. S., SNODGRASs R. T.: Temporal data management. IEEE TKDE 11, 1 (1999), 36-44. 3

[JS09] JIN J., SZEKELY P.: Querymarvel: A visual query language for temporal patterns using comic strips. In Procs. VL/HCC (Sept 2009), pp. 207-214. 3

[JS10] JIN J., SZEKELY P.: Interactive querying of temporal data using a comic strip metaphor. In Procs. VAST (2010), pp. 163-170. 3

[Kar94] KARAM G. M.: Visualization using timelines. In ACM SIGSOFT (1994), pp. 125-137. 3

[KSF*02] Kent W. J., Sugnet C. W., Furey T. S., Roskin K. M., Pringle T. H., ZAhler A. M., HAussler D.: The human genome browser at ucsc. Genome Research 12, 6 (2002), 996-1006. 3

[MCD17] Miao H., Chavan A., Deshrande A.: Provdb: Lifecycle management of collaborative analysis workflows. In HILDA@SIGMOD (2017), pp. 7-1. 3
[MLDD17] Miao H., Li A., Davis L. S., Deshrande A.: Modelhub: Deep learning lifecycle management. In IEEE 33rd International Conference on Data Engineering (ICDE) (2017), pp. 1393-1394. 3

[MLMdO*13] Monroe M., Lan R., Morales del Olmo J., ShneiderMAN B., Plaisant C., Millstein J.: The challenges of specifying intervals and absences in temporal queries: A graphical language approach. In Procs. CHI (2013), pp. 2349-2358. 3

[PMR* 96] Plaisant C., Milash B., Rose A., WidofF S., Shneiderman B.: Lifelines: visualizing personal histories. In Proceedings of the SIGCHI conference on Human factors in computing systems (1996), ACM, pp. 221-227. 3

[RAL*17] Ren D., Amershi S., LeE B., SuH J., Williams J. D.: Squares: Supporting interactive performance analysis for multiclass classifiers. IEEE TVCG 23 (2017), 61-70. 1, 3

[RBER17] RATNER A. J., BACH S. H., EHRENBERG H. R., RÉ C.: Snorkel: Fast training set generation for information extraction. In Proceedings of the 2017 ACM International Conference on Management of Data (2017), ACM, pp. $1683-1686.3$

[RDSW*16] RATner A. J., De Sa C. M., Wu S., Selsam D., RÉ C.: Data programming: Creating large training sets, quickly. In Advances in Neural Information Processing Systems (2016), pp. 3567-3575. 3

[RTW*11] ROBINSON J. T., THORVALDSDOTTIR H., WINCKLER W., Guttman M., Lander E. S., Getz G., Mesirov J. P.: Integrative genomics viewer. Nature Biotechnology 29, 1 (2011), 24-26. 3

[SAA*94] SnOdgrass R. T., Ahn I., Ariav G., BATORY D., ClifFord J., Dyreson C. E., Elmasri R., Grandi F., JENSEN C. S., KÄFER W., Kline N., KUlKarni K., LEUnG T. Y. C., LORENTZOS N., Roddick J. F., SEgEV A., Soo M. D., SRIPADA S. M.: Tsq12 language specification. SIGMOD Rec. 23, 1 (1994), 65-86. 3

[SGM*04] Stalker J., Gibiins B., Meidl P., Smith J., Spooner W. Hotz H.-R., Cox A. V.: The ensembl web site: Mechanics of a genome browser. Genome Research 14, 5 (2004), 951-955. 3

[SHG*15] Sculley D., Holt G., Golovin D., Davydov E., Phillips T., Ebner D., Chaudhary V., Young M., Crespo J.-F., Dennison D.: Hidden technical debt in machine learning systems. In Advances in Neural Information Processing Systems (2015), pp. 2503-2511. 3

[SMWH17] SATYANARAYAN A., MORITZ D., WongSuphasaWAT K., HEER J.: Vega-lite: A grammar of interactive graphics. IEEE Transactions on Visualization and Computer Graphics 23, 1 (2017), 341-350. 3

[STH02] Stolte C., TANG D., HANRAhan P.: Polaris: a system for query, analysis, and visualization of multidimensional relational databases. IEEE TVCG 8, 1 (2002), 1-14. 3

[vdWPS*17] VAN DER WEIDE T., PAPAdOPOUlOS D., SMIRnOV O. ZIELINSKI M., VAN KASTEREN T.: Versioning for end-to-end machine learning pipelines. In Proc. the 1st Workshop on Data Management for End-to-End Machine Learning (2017). 3

[VSL*16] VARTAK M., Subramanyam H., LEE W.-E., VisWanathan S., Husnoo S., Madden S., Zaharia M.: ModelDB: a system for machine learning model management. In Proc. HILDA (2016). 3

[WGGP*] Wongsuphasawat K., Guerra Gómez J. A., Plaisant C., WANG T. D., TAIEB-Maimon M., ShNeIderman B.: Lifeflow: Visualizing an overview of event sequences. In Procs. CHI, pp. 1747-1756. 3

[Wil05] Wilkinson L.: The Grammar of Graphics (Statistics and Computing). Springer-Verlag, 2005. 3

[ZDFD15] Zgraggen E., Drucker S. M., Fisher D., DeLine R.: (s|qu)eries: Visual regular expressions for querying and exploring event sequences. In Procs. CHI (2015), ACM. 3 